\title{
Timeline representation of clinical data: usability and added value for pharmacovigilance
}

Thibault Ledieu ${ }^{1,2^{*}}$ (D) Guillaume Bouzillé ${ }^{1,2,3}$, Frantz Thiessard ${ }^{4}$, Karine Berquet ${ }^{5}$, Pascal Van Hille ${ }^{1,2,3}$, Eric Renault ${ }^{1,2}$, Elisabeth Polard ${ }^{5}$ and Marc Cuggia ${ }^{1,2,3}$

\begin{abstract}
Background: Pharmacovigilance consists in monitoring and preventing the occurrence of adverse drug reactions (ADR). This activity requires the collection and analysis of data from the patient record or any other sources to find clues of a causality link between the drug and the ADR. This can be time-consuming because often patient data are heterogeneous and scattered in several files. To facilitate this task, we developed a timeline prototype to gather and classify patient data according to their chronology. Here, we evaluated its usability and quantified its contribution to routine pharmacovigilance using real ADR cases.
\end{abstract}

Methods: The timeline prototype was assessed using the biomedical data warehouse eHOP (from entrepôt de données biomédicales de l'HOPital) of the Rennes University Hospital Centre. First, the prototype usability was tested by six experts of the Regional Pharmacovigilance Centre of Rennes. Their experience was assessed with the MORAE software and a System and Usability Scale (SUS) questionnaire. Then, to quantify the timeline contribution to pharmacovigilance routine practice, three of them were asked to investigate possible ADR cases with the "Usual method" (analysis of electronic health record data with the DxCare software) or the "Timeline method". The time to complete the task and the data quality in their reports (using the vigiGrade Completeness score) were recorded and compared between methods.

Results: All participants completed their tasks. The usability could be considered almost excellent with an average SUS score of $82.5 / 100$. The time to complete the assessment was comparable between methods $(P=0.38)$ as well as the average vigiGrade Completeness of the data collected with the two methods $(P=0.49)$.

Conclusions: The results showed a good general level of usability for the timeline prototype. Conversely, no difference in terms of the time spent on each ADR case and data quality was found compared with the usual method. However, this absence of difference between the timeline and the usual tools that have been in use for several years suggests a potential use in pharmacovigilance especially because the testers asked to continue using the timeline after the evaluation.

Keywords: Informatics, Information visualization, Pharmacovigilance, Usability testing

\footnotetext{
* Correspondence: thibault.ledieu@gmail.com

${ }^{1}$ INSERM, U1099, F-35000 Rennes, France

${ }^{2}$ Université de Rennes 1, LTSI, F-35000 Rennes, France

Full list of author information is available at the end of the article
}

(c) The Author(s). 2018 Open Access This article is distributed under the terms of the Creative Commons Attribution 4.0 International License (http://creativecommons.org/licenses/by/4.0/), which permits unrestricted use, distribution, and reproduction in any medium, provided you give appropriate credit to the original author(s) and the source, provide a link to the Creative Commons license, and indicate if changes were made. The Creative Commons Public Domain Dedication waiver (http://creativecommons.org/publicdomain/zero/1.0/) applies to the data made available in this article, unless otherwise stated. 


\section{Background}

Pharmacovigilance is defined by the World Health Organization (WHO) as "the science and activities relating to the detection, assessment, understanding and prevention of adverse effects and any other drug-related problem." [1] Pharmacovigilance requires the retrospective collection of information from the patients' medical records in order to find clues concerning drug accountability in the occurrence of adverse drug reactions (ADR). This means finding relevant information in patient records that may be evidence of an ADR. Clinical data are heterogeneous in terms of structure (structured or textual data) and domain (e.g., clinical/laboratory results or prescription data). Searching and organizing such information require specific expertise and is often a time-consuming task due to the lack of adapted tools for this purpose.

The re-use of clinical data from patients has been facilitated by the emergence of querying interfaces for clinical data warehouses, such as i2b2 tools [2] or complete systems (database and interface), such as eHOP [3]. These systems allow querying and analysing heterogeneous clinical data, and have been successfully exploited for clinical research (e.g., feasibility studies, patients' pre-screening and recruitment for clinical trials [4]) and epidemiological studies (cohort detection) [5]. Pharmacovigilance is another field where the exploitation of health databases could play a key role [6].

Among the different data-mining methods, visual data analysis could be useful for exploring complex data. Indeed, visualization of data on the history of individual patients or groups of patients could allow highlighting chronological clues and causal relationships between drug exposure and ADR. A timeline is an interactive chronological representation of a list of events that can include different types of data and time scales. In the literature, several timeline tools for electronic health record (EHR) data visualization to explore individual or population-wide clinical data (e.g., Lifeline [7], Outflow [8], VisuExplore [9], Eventflow [10]) have been described. For instance, CareCruiser [11] provides simultaneously a view of the patient's data and therapeutic protocols for the analysis of treatment responses (e.g., for a patient on oxygen, the $\mathrm{O}_{2}$ saturation values will vary depending on the treatment). VisuExplore [9] offers different types of graphs (distribution curves, histograms) depending on the data type (e.g., for a diabetic patient, insulin administration is displayed as dots, while biological parameters, such as glucose concentration, are displayed as distribution curves). KNAVE-II [12] is an interactive and semantic viewer of clinical data based on the use of domain ontologies. However, none of these tools has been evaluated for pharmacovigilance.

This work focused on a timeline tool developed in the framework of the Retrieval and Visualization of Electronic Health Records (RAVEL) [13] and Pharmaco-Epidemiology of Health Products (Pharmaco-Epidémiologie des Produits de Santé, PEPS) research projects the main mission of which is the development of new methods for information research and visualization of heterogeneous clinical data. In this paper we present the evaluation of the usability of this prototype timeline and the quantification of its contribution to pharmacovigilance routine practice in terms of time saving and quality of the collected data.

\section{Implementation \\ Timeline description}

The prototype's graphical user interface (GUI) was developed using the JavaScript d3.js library [14]. For a broad use, this component was designed as a web service, weakly coupled to the EHR data source (eHOP for the prototype). eHOP (from entrepôt de données biomédicales de l'HOPital), the biomedical data warehouse used by Rennes University Hospital Centre, contains comprehensive laboratory and clinical data on 1.6 million patients. It provides access to heterogeneous clinical information in the form of free text (hospitalization reports, discharge letters, etc.), of data coded using different terminologies (e.g., the International Classification of Diseases, 10th revision, ICD-10, medical diagnosis codes), and structured data (laboratory results, prescriptions and drug administrations). The prototype used a web interface to allow interactive navigation. Real patient data were used for the prototype assessment. Figure 1 is a screen mock-up in English to show how the information is displayed in the timeline. A real screenshot (in French) and demo are available in Additional file 1.

To the best of our knowledge, this application includes new features. Specifically, semantic data aggregation/expansion was implemented by leveraging the hierarchical properties of different terminologies (Anatomical Therapeutic Chemical, ATC, classification system; French Common Classification of Medical Procedures, CCAM; ICD-10; and the local terminology used by the Clinical Laboratory of Rennes). This approach allows users to interactively control the granularity of the displayed information.

Medical codes (e.g., ICD-10 codes) can be directly searched in different terminologies using a hierarchical tree. This tree also allows users to choose the structured data they wish to display in the timeline. The choice of concepts to display can be pre-set according to the user's specific medical field. The user also can search for terms in text documents via a free text search field. Each type of data has its own representation mode: graphs for clinical test results, windows for textual records and iconic language for medical terminologies (Visualization of Concepts in Medicine icons) [15]. The user can expand or reduce the time scale at will through two navigation controls: the overall time period selection and the timeline overview. 


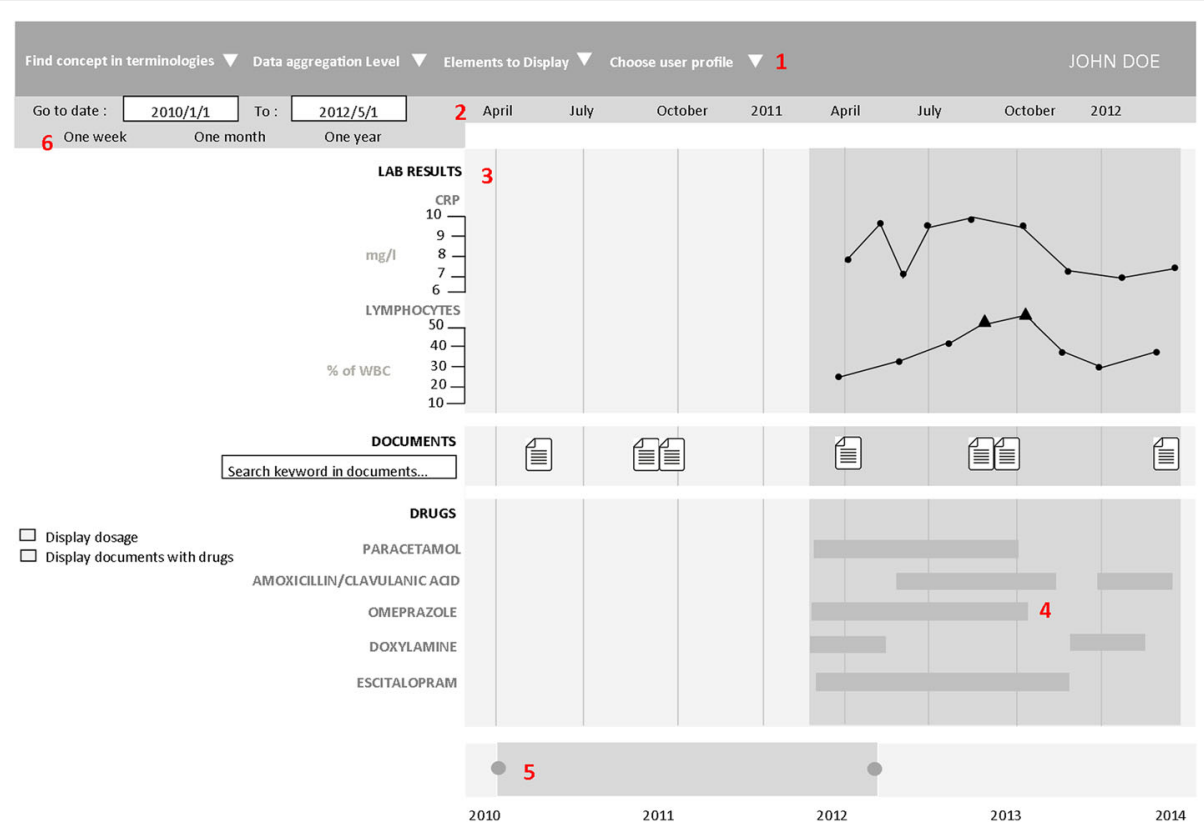

Fig. 1 Timeline interface. The interface individual components are: 1. Selection of patient, laboratory and clinical data and medical codes (e.g., ICD-10 code). 2. Time scale. 3. Laboratory results in the form of graphic curves (dots show normal values; triangles and their orientation represent anomalous values). 4. Drug prescriptions and treatment duration (start, end and overall duration time, as well as the drug regimen. 5. Timeline overview for navigation. 6 . Overall time period selection 4.

All these features were designed to facilitate navigating through the complexity of patient data.

\section{Recruitment of experts}

Expert users were recruited from a team of pharmacists and physicians who work at the Regional Pharmacovigilance Centre in Rennes and who are part of the target audience for our tool. Based on literature data [16], it was determined that five users were sufficient to identify $80 \%$ of the problems linked to the application's usability [17]. Finally, six people (five women and one man among whom there were five pharmacists and one physician) could be recruited for the usability study, and three of them were also involved in the impact quantification study. These participants perform routinely pharmacovigilance activities. The six people received no financial compensation in exchange for their participation. Their mean age was $31.3 \pm 4.5$ years (range: 25 to 40 ). All routinely used computers as part of their pharmacovigilance activity, particularly to study patient records.

\section{Usability evaluation method}

Usability is defined by the International Organization for Standardization (ISO) as the "extent to which a product can be used by specified users to achieve specified goals with effectiveness, efficiency and satisfaction in a specified context of use". [18] There are many methods for evaluating usability [17] and several are used for the development and evaluation of health-related software programs [19-21]. We chose the 'think-aloud protocol' approach that has been described by Nielsen as "the most reliable evaluation method to obtain a usability estimate" [22]. It is based on recording the participants' opinion while they perform a series of tasks designed specifically for the usability evaluation [23]. Participants are asked to orally state their thoughts, perceptions and opinions while interacting with the application (concurrent think aloud method). The participants' performance during the execution of these tasks is then analysed by the evaluator. This method can be applied throughout all stages of the web application development and provides qualitative data that will help evaluators to identify usability problems. It can also prompt the development of new features for the interface in response to the users' feedback. The severity of the usability problems encountered by users was rated with the Nielsen rating scale (from $0=$ No usability problem to $4=$ Usability catastrophe) [24]. Peute and al. [25] showed that compared with the retrospective think aloud method, the concurrent method increases participants' verbalization and leads to a higher detection rate of usability problems. In addition, this method seems to be more reliable than the retrospective analysis of the test record, conducted in the users' presence.

\section{Usability evaluation use case}

An ADR case, chosen in collaboration with the head of the Pharmacovigilance Centre, was used to test all the 
prototype's features in a real-life situation. Participants were asked to determine whether or not the adverse reaction experienced by a patient admitted in intensive care resulted from exposure to an antibiotic. To this aim, they had to perform four main tasks: i) to find the patient's name and address, ii) to determine the exact nature of the adverse reaction and collect all pertinent information, iii) to find information on the suspected drug (name and dose, for instance) and iv) to collect information on the ADR outcome.

\section{Usability evaluation procedure}

Before the usability test, all users underwent, at the same time, training to become familiar with the timeline functionalities for about one hour. For this training session, fictitious patient data were created in the same format as the actual data to be able to show/test the features of the timeline without having to access real patient data. During this training session, participants could visualize the data of a fictitious patient in the timeline (e.g., drug prescriptions, laboratory data, reports) and could use each functionality at least once (see Timeline description chapter for details on these functions). If a user encountered a problem, the trainer helped him/her to overcome this problem. This training session and the usability test of the prototype took place at the Medical Informatics Laboratory of Rennes University. The working environment (operating system, computers, etc.) was the same as the one used at the Pharmacovigilance Centre. Usability tests (six sessions with one participant per session) were performed in the presence of the evaluator, who observed the interactions between participant and interface and noted his/her observations on paper. Each participant received a notebook to record all relevant data retrieved on the use case. The usability testing sessions were recorded on video, as well as all interactions with the interface (keyboard input and mouse movements within the application). The recording and subsequent analysis were made using MORAE (TechSmith, software version 3.3.4). The video recordings were analysed after all participants had completed all the tasks. The MORAE software can capture on-screen video footage, while simultaneously recording the interactions between participant and GUI. Moreover, the participants' face was also recorded, thus allowing the evaluators to assess, retrospectively, their expression and reactions while interacting with the prototype.

At the end of the usability test, each participant filled in the System and Usability Scale (SUS), translated into French. This questionnaire allows measuring the application's usability in a fast and reliable way [26], and scoring was done as proposed by Sauro and Lewis. [27] Finally, each participant was interviewed using open-ended questions to determine the timeline features they most appreciated and whether they encountered any particular problems. In addition, users were asked about suggestions to improve the timeline. The interviews were not recorded.

\section{Quantitative impact study}

For the impact study, the timeline prototype was slightly modified to take into account the suggestions/remarks from the six participants after the usability test (the added features are described in the Results section).

\section{Selection of patient records for the impact study}

For the quantitative impact study, 743 potential cases from the 2015 eHOP data were selected according to a list of ICD-10 codes that were potentially related to ADR and the method described by Osmont et al. [28]. This list was defined in a previous internal study of the Pharmacovigilance Centre based on 2014 data. Only ICD-10 codes with the highest probability of matching an ADR were selected (Fig. 2). Then, 85 cases were randomly chosen, in order to have roughly the same proportion of the different ICD-10 codes for the three participants and for each analysis method (usual method versus timeline, see below). A Python script was used to randomize the cases among the three participants.

\section{Procedure for impact quantification}

These 85 cases were reviewed by three members of the Regional Pharmacovigilance Centre of Rennes using two methods: the "usual method" (EHRs and DxCare software, see Additional files 2 and 3), and the "timeline" method (timeline prototype). Participants were provided with a list of patient records and instructions on which method to use for each record (Fig. 2). The working environment (operating system, computers, etc.) was the same as the one used at the Pharmacovigilance Centre. As in the routine pharmacovigilance practice, they had to gather information to establish a possible causality link between the diagnosis and the administration of a drug. They also wrote down the start time and end time for processing each file. The process was considered finished when the participant had retrieved all the required information or when he/ she considered that this information was not present or could not be found with the used method.

After reviewing a case, participants filled in a homemade questionnaire detailing the following information: complexity of the case, information about the ADR, which functions of the timeline were used, and general comments.

The report quality was measured using a method based on the WHO vigiGrade Completeness score that includes quality indicators, such as the drug name or description of the ADR (see Additional file 4). Furthermore, absence of 


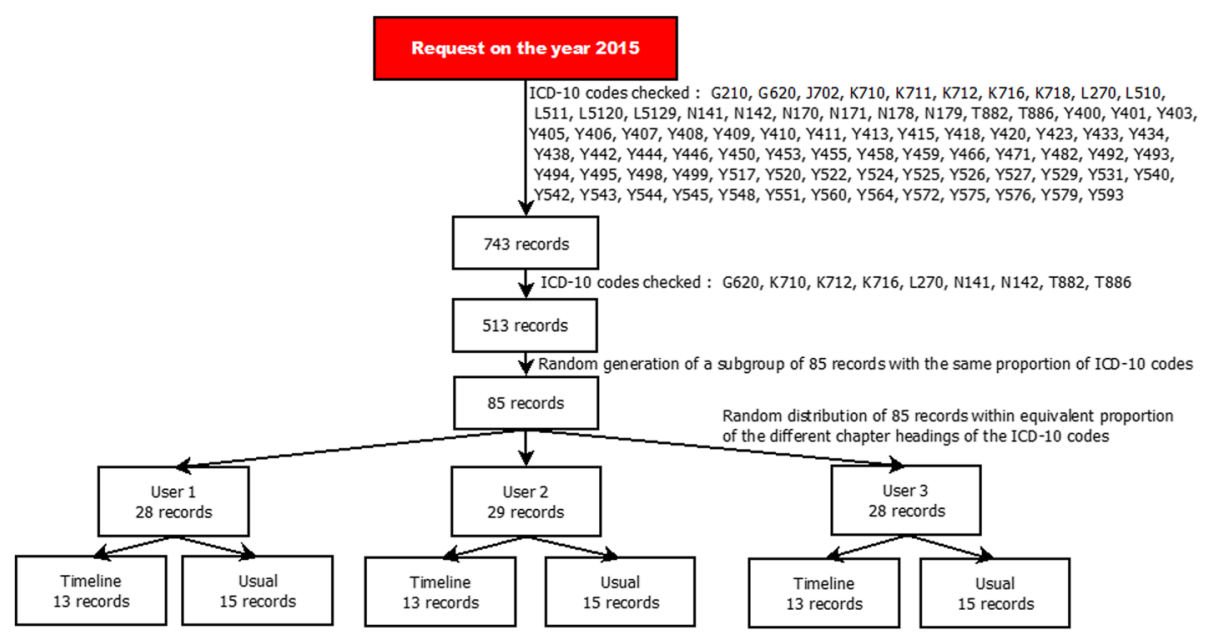

Fig. 2 Randomization of patient records 8

information affected the resulting score. A member of the Pharmacovigilance Centre not involved in the timeline testing did the scoring.

All analyses were conducted on the 85 cases, but for the vigiGrade Completeness score comparison that concerned only the cases considered to be ADR by the participants. Time differences between methods were also compared according to the participants and according to the chapter of the ICD-10 codes.

\section{Statistical analyses}

Quantitative variables were expressed as mean and standard deviation. Continuous variables were compared using the Student's $t$ test or Wilcoxon rank-sum test, as appropriate, for two groups, and with the Kruskal-Wallis test for more than two groups. $P$ values $<0.05$ were considered significant. Analyses were performed with the $\mathrm{R}$ statistical software, version 3.2.2. [29]

\section{Results}

\section{Usability evaluation}

The participants' average SUS score was 82.5 out of 100 points. After responding to the questionnaire, all participants expressed the desire to use the timeline in their daily practice.

All participants could successfully perform the tasks required for the use case. The use case completion time varied greatly among participants (mean completion time $=24.44 \pm 9.97 \mathrm{~min}$ ). The click count also was variable among participants (mean number of clicks: $174 \pm$ 92.41, $P=0.06)$.

The error distribution in handling the interface did not vary much among participants (mean number of errors: $1.33 \pm 1.5)$. The task to gather information on the ADR outcome had the highest error count $(P=0.16)$.

Table 1 lists the usability issues encountered by participants during the test. The severity of each

Table 1 Usability issues

\begin{tabular}{|c|c|c|c|c|}
\hline Problem & Source of the problem & $\begin{array}{l}\text { Problem } \\
\text { frequency }\end{array}$ & $\begin{array}{l}\text { Nielsen scale } \\
\text { score }\end{array}$ & Observations \\
\hline $\begin{array}{l}\text { Zoom increase/decrease functionality } \\
\text { deemed } \\
\text { insufficiently accurate by participants }\end{array}$ & User Interface (UI) & $6 / 6(100 \%)$ & $3 / 4$ & $\begin{array}{l}\text { The Timeline overview does not allow accurate } \\
\text { zooming to select the detailed view range }\end{array}$ \\
\hline Absence of prescription data & Data source & $6 / 6(100 \%)$ & $3 / 4$ & $\begin{array}{l}\text { Prescriptions were not digitized in the Intensive } \\
\text { Care Unit }\end{array}$ \\
\hline $\begin{array}{l}\text { Text highlighting did not work during } \\
\text { the } \\
\text { search }\end{array}$ & UI & $1 / 6(17 \%)$ & $3 / 4$ & Issues with accent detection \\
\hline Medication regimens were not displayed & UI & $6 / 6(100 \%)$ & $3 / 4$ & Data on medication regimens were not available \\
\hline $\begin{array}{l}\text { Hierarchical tree of biological concepts: } \\
\text { navigation problems }\end{array}$ & $\begin{array}{l}\text { Complexity of the } \\
\text { terminology in the UI }\end{array}$ & $3 / 6(50 \%)$ & $2 / 4$ & $\begin{array}{l}\text { The hierarchical tree of laboratory results was too } \\
\text { large to allow users to easily find information } \\
\text { without filtering }\end{array}$ \\
\hline Repositioning document windows & UI bug & $2 / 6(34 \%)$ & $2 / 4$ & The window slid under the menu bar \\
\hline
\end{tabular}


usability problem was evaluated with the Nielsen rating scale $[24]$.

\section{Corrections / adjustments of the application}

The usability assessment allowed users to make recommendations for improving the user interface. These recommendations have led us to adding new features designed specifically for pharmacovigilance, such as the option to automatically extract the drug names from textual documents and the ability to access, directly from the timeline, a module for visualizing the drug monography.

\section{Evaluation of the application quantitative impact on the routine practice}

Three participants (two pharmacists and one physician) were recruited for this evaluation. All were also involved in the usability study.

Overall, the time required for task completion was comparable between methods (16 $29^{\prime \prime}$ and $15^{\prime} 51^{\prime \prime}$ for the usual method and the timeline, respectively; $P=0.38$ ) (Fig. 3).

Comparison among the three participants, showed that for user 1 , completion time was significantly lower with the timeline than with the usual method $(P=0.02)$. No difference between methods was observed for the other two participants (Table 2 and Fig. 4).

Finally, the completion time was compared between methods and the type of ICD-10 chapter (Table 3). Although not significantly different, completion time tended to slightly faster with the timeline method than the usual method only for cases concerning genitourinary system diseases $(P=0.08)$. This difference could be explained by the systematic need to consult the patient's laboratory results to judge the drug involvement in this type of ADR.

Among the 85 selected cases, 68 corresponded to actual ADR. Therefore, data quality was assessed and compared only for these records using the vigiGrade Completeness score. Overall, the average quality of the notifications (report for each adverse event) produced with the two methods was not significantly different $(P=0.49)$. Similar results were obtained also when comparing the notification quality among the three participants (Table 4).

\section{Participants' feedback}

During the interviews participants expressed the wish to use the timeline in their routine practice. The most appreciated features were the search functionality in the text and prescription display. The interviews also highlighted some negative points: problems in choosing the range of time of the timeline and difficulties in selecting the elements to display in the timeline. Moreover, the participants wished to see the drug regimens in addition to the prescriptions.

After the evaluation of the timeline impact on the pharmacovigilance practice, participants reiterated their interest in obtaining the application for use in their daily practice. They stated that the timeline allowed them to save time in all cases that required consulting laboratory results. Moreover, they added that the possibility to display data prescription and information on drug

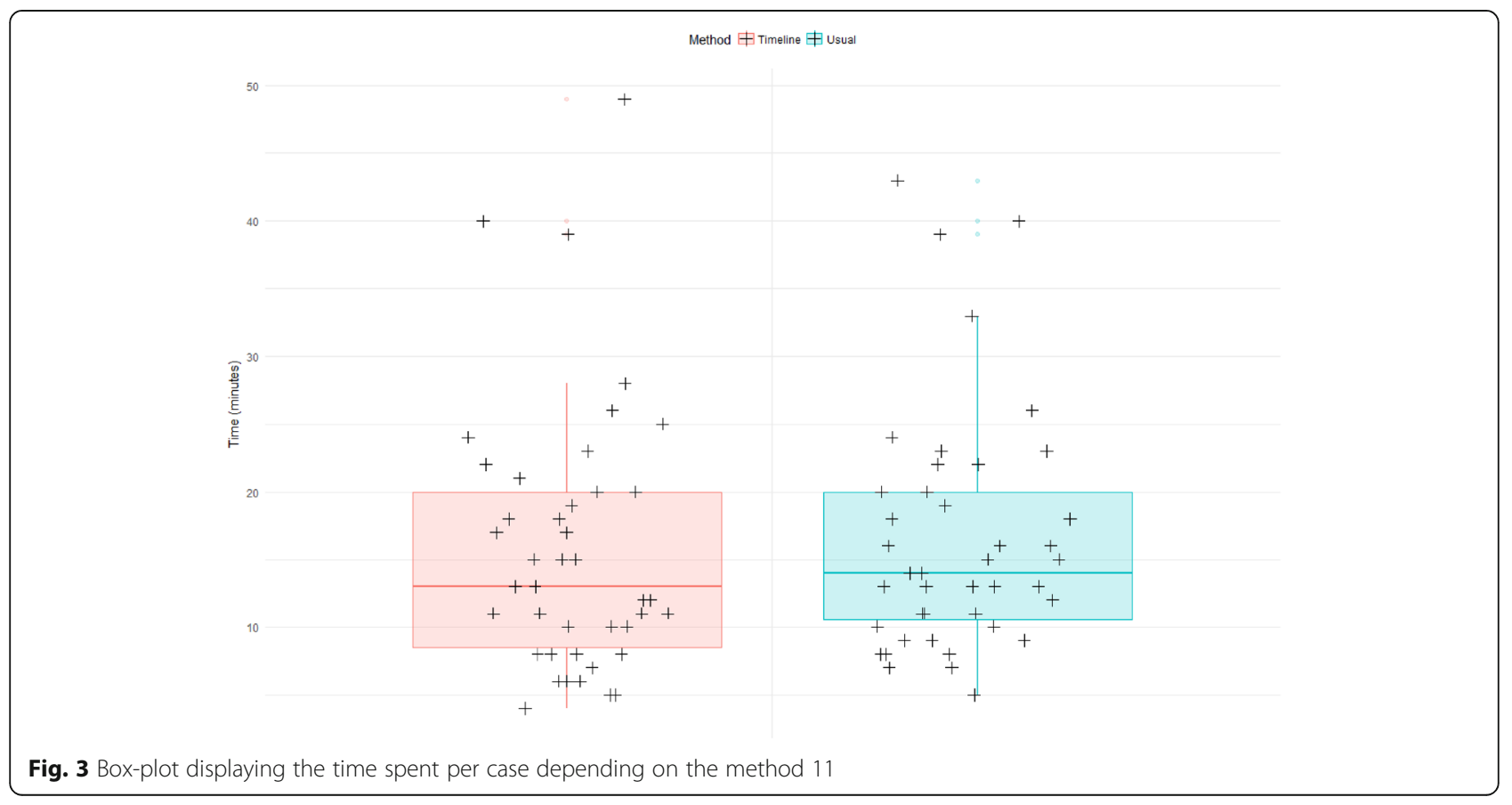


Table 2 Average time spent per case in function of the method and the participant

\begin{tabular}{lllll}
\hline Method Participant & Usual & Timeline & Difference & $P$-value \\
\hline Participant 1 & $17^{\prime} 26^{\prime \prime}$ & $11^{\prime} 38^{\prime \prime}$ & $-5^{\prime} 48^{\prime \prime}$ & 0.02 \\
Participant 2 & $15^{\prime} 55^{\prime \prime}$ & $18^{\prime} 11^{\prime \prime}$ & $+2^{\prime} 16^{\prime \prime}$ & 0.71 \\
Participant 3 & $16^{\prime} 8^{\prime \prime}$ & $17^{\prime} 40^{\prime \prime}$ & $+1^{\prime} 32^{\prime \prime}$ & 0.59 \\
\hline
\end{tabular}

administration in a chronological format was a considerable advantage.

\section{Discussion}

\section{Usability evaluation}

The results of the timeline usability test were almost excellent, as indicated by the SUS score (82.5) and users' feedback. The SUS questionnaire score indicates a good usability level. This score places the timeline in the $10 \%$ of applications with usability scores higher than $80 \%$, [30] and is higher than that of the KNAVE-II tool (69.1) [31]. The reported usability problems were, in general, minor defects that could be rapidly corrected. Moreover, based on the users' feedback, new functionalities were introduced to better meet the needs of pharmacovigilance users. The significantly higher number of errors during the task of searching the ADR outcome can be explained by the high complexity of this task (search involving laboratory results, reading several documents simultaneously, etc.). Differences in completion times can be explained by differences in each participant's working method. Some participants carried out their tasks in parallel, while others performed them one after the other. This explanation also applies to the difference in the number of clicks, because some participants had to search for the same information more than once.

\section{Evaluation of the application quantitative impact on the routine practice}

The evaluation of the timeline quantitative impact on the routine practice showed a significant gain of time for one of the participants. This user was the one who participated the most in the development of the application and had the least experience with the usual method, compared with the others. This observation suggests that the gain of time could have been more general with a longer preliminary training session to better familiarize all participants with the timeline functionalities. Moreover, as the timeline was compared to the method that has been used in the Pharmacovigilance Centre for more than 10 years, the overall absence of time-saving supports the need for a new evaluation after participants have become more accustomed to the timeline tool, especially because the users wanted to be able to continue using the timeline after the evaluation.

Overall, for simple cases for which retrieving the record of the hospital stay is sufficient, the timeline added value is less apparent.

Finally, the absence of any significant difference in the quality of reporting shows that the exclusive use of the timeline is not a limiting factor for data collection. Moreover, for some cases processed using the timeline, the poor quality of reporting could be explained by the presence of only textual documents in the application. This lack of data was not related to the timeline itself, but to the few data available in the data warehouse.

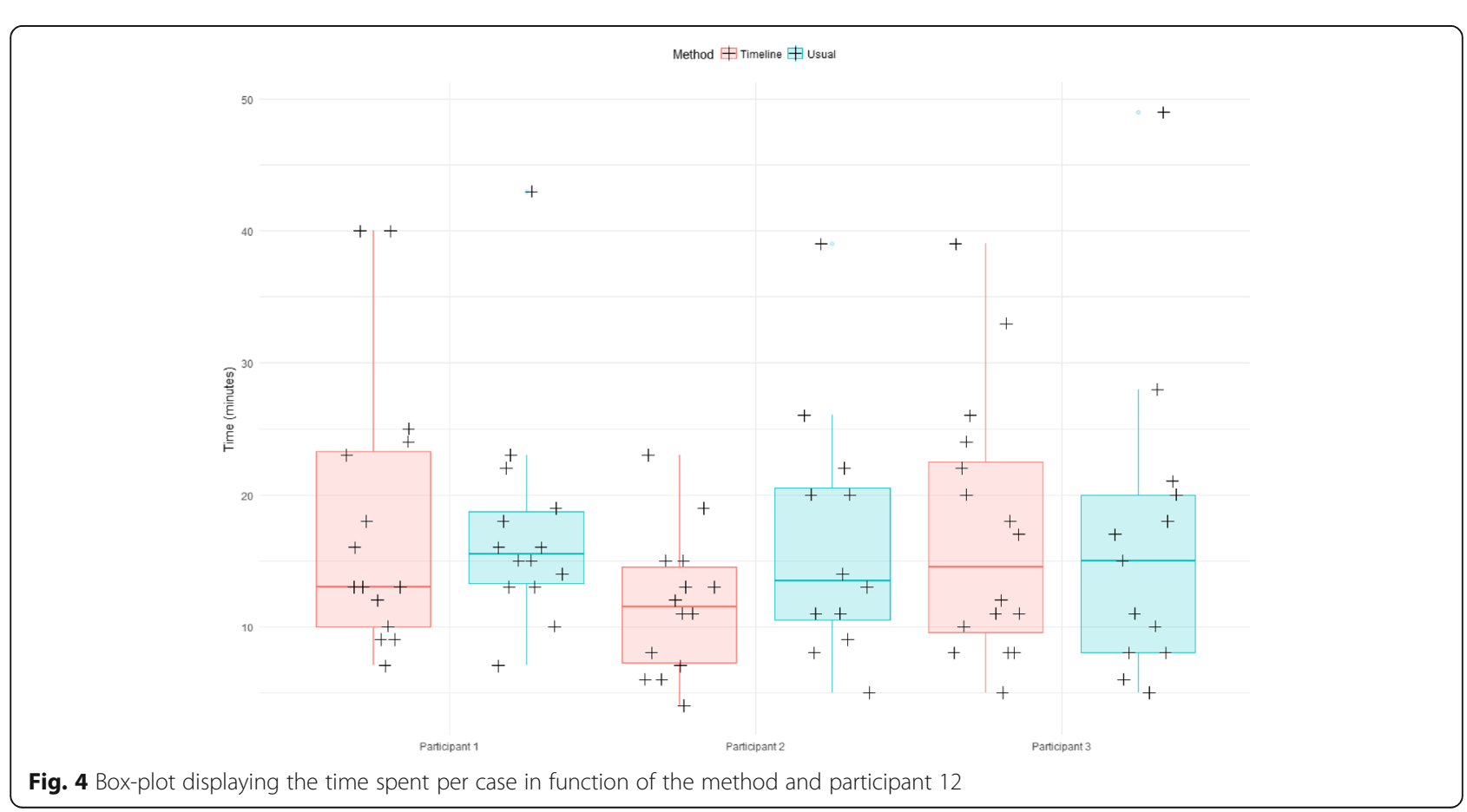


Table 3 Average time spent per case in function of the method and ICD-10 chapter

\begin{tabular}{|c|c|c|c|}
\hline ICD-10 Chapter & Usual (n) & Timeline (n) & Difference \\
\hline XIV - Diseases of the genitourinary system & $17^{\prime} 36^{\prime \prime}[20]$ & $13^{\prime} 10^{\prime \prime}[18]$ & $-4^{\prime} 26^{\prime \prime}$ \\
\hline XII - Diseases of the skin and subcutaneous tissue & $15^{\prime} 50^{\prime \prime}[12]$ & $15^{\prime} 20^{\prime \prime}[9]$ & $-30 "$ \\
\hline$X X$ - External causes of morbidity and mortality & $15^{\prime} 17^{\prime \prime}[7]$ & $15^{\prime} 15^{\prime \prime}[4]$ & $-2^{\prime \prime}$ \\
\hline $\mathrm{Vl}$ - Diseases of the nervous system & $17^{\prime}[5]$ & $20^{\prime}[9]$ & $+3^{\prime}$ \\
\hline$X I$ - Diseases of the digestive system & $13^{\prime}$ & $17^{\prime} 45^{\prime \prime}$ & $+4^{\prime} 45^{\prime \prime}$ \\
\hline $\begin{array}{l}\text { XIX - Injury, poisoning and certain other consequences } \\
\text { connected with external causes }\end{array}$ & $12^{\prime} 40^{\prime \prime}$ & $14^{\prime} 12^{\prime \prime}$ & $+1^{\prime} 32^{\prime \prime}$ \\
\hline
\end{tabular}

\section{Future versions}

On the basis of the results and feedback obtained in this study, we are planning to introduce new features in the next version of the application. Particularly, we will focus on better displaying textual records, which is the starting point for locating information within patient records. Also, it will be possible to more easily compare the contents of a document with the structured data displayed in the timeline.

\section{Limits of the study}

During the usability test, breaking down the use case into tasks caused issues in the analysis of the MORAE records. Indeed, these tasks did not occur sequentially, but in parallel because in pharmacovigilance the investigation process does not follow a single critical path. In addition, for the same task, participants did not search the same level of details. Some participants wanted very specific and detailed information (e.g., the half-life of the molecule administered to the patient), and therefore they required more time to complete their tasks. Conversely, other participants simply used the information contained in the text document retrieved by the query. It is, therefore, difficult to attribute the time differences observed during the execution of different tasks to an application usability problem.

For the impact study, only three participants were available, due to the limited human and material resources at our disposal, and therefore, it was not possible to process the same record twice using the two methods. A more thorough comparison between methods would require greater test power.

Table 4 Mean notification quality according to the method and the user

\begin{tabular}{llll}
\hline Participant & $\begin{array}{l}\text { Average quality, } \\
\text { usual method }\end{array}$ & $\begin{array}{l}\text { Average quality, } \\
\text { timeline }\end{array}$ & $\begin{array}{l}\text { Overall average } \\
\text { quality }\end{array}$ \\
\hline Participant no. 1 & 0.87 & 0.81 & 0.84 \\
Participant no. 2 & 0.81 & 0.85 & 0.84 \\
Participant no. 3 & 0.81 & 0.74 & 0.78 \\
Total & 0.83 & 0.80 & 0.81 \\
\hline
\end{tabular}

\section{Conclusion}

The complexity and richness of data found in medical records require the development of tools for their efficient exploitation. Among the required features, viewing and querying information within a file remain critical elements. As part of the RAVEL project, we developed an integrated method for searching and visualizing information, in a timeline format. This article details the evaluation of this timeline. The results of the usability test indicate a good general level of usability, and new features adapted to pharmacovigilance routine practice could be added on the participants' request. The timeline is now available to people in charge of pharmacovigilance for their daily practice.

\section{Availability and requirements}

Project name: RAVEL Timeline.

Project home page: e.g. http://devtools.univ-rennes1.fr/ websvn/Timeline/

Operating system(s): Platform independent.

Programming language: Java 8, HTML 5, CSS 3, JavaScript.

Other requirements: Java 8 or higher, Oracle $11 \mathrm{~g}$ or higher.

License: GNU GPL.

Any restrictions to use by non-academics: For use by non-academics, please contact Pr Marc Cuggia: marc.cuggia@univ-rennes1.fr

\section{Additional files}

Additional file 1: Timeline interface (original French version). The interface individual components are: 1 . Selection of patient, laboratory and clinical data and medical codes (e.g., ICD-10 code). 2. Time scale. 3. Laboratory results in the form of graphic curves (green dots are normal values, triangles and their orientation correspond to anomalous values). 4. Drug prescriptions and treatment duration (start, end and overall duration) as well as the drug regimen. 5. Timeline overview for navigation. 6. Overall time period selection. (PNG $140 \mathrm{~kb}$ )

Additional file 2: Portfolio interface (in French) (PNG 392 kb)

Additional file 3: Portfolio mock-up (PNG 63 kb)

Additional file 4: Dimensions included in the vigiGrade Completeness score and the corresponding penalties. (PNG $153 \mathrm{~kb}$ ) 


\section{Abbreviations}

ADR: Adverse drug reaction; ATC: Anatomical therapeutic chemical terminology; CCAM: French classification, for the medical diagnostic or therapeutic procedures (Classification Commune des actes médicaux): eHOP: Biomedical Data Warehouse of Hospital (Entrepôt de données biomédicales de l'HOPital); EHR: Electronic health record; ETL: Extract transform load; GUI: Graphical user interface; HMI: Human-machine interface; ICD-10: International classification of diseases, 10th edition; PEPS: Pharmacoepidemiology health products (Pharmaco-Epidémiologie des Produits de Santé); RAVEL: Retrieval and visualization in electronic health records; SUS: System usability scale; UI: User interface; VCM: Visualization of medical knowledge (Visualisation des Connaissances Médicales); WHO: World Health Organization

\section{Acknowledgements}

We thank Dr. Catherine Plaisant, Associate Director of Research of the HumanComputer Interaction Laboratory for her advices in the methodological choices and her attentive reading of the manuscript. We also thank Dr. Elisabetta Andermarcher for her careful proofreading of the paper. We also thank Dr. Jean-Baptiste Lamy for agreeing to provide us the VCM language.

\section{Funding}

This work was supported by the French National Research Agency (ANR) (Project ID: ANR-11-TECS-0012) and the PEPS consortium (Pharmaco-Epidemiology of Health Products consortium).

\section{Availability of data and materials}

Data on the demo and screenshot are dummy data. Data used for the evaluation came from our clinical data warehouse, eHOP. [3] The main author works at the Clinical Data Centre of Rennes CHU and it is within this framework that he had access to these data.

\section{Consent to publish}

Not applicable.

\section{Authors' contributions}

$T L, E R$, and $P V H$ developed and implemented the prototype of the timeline. $T L, G B, K B$ and $M C$ defined the study methodology. TL, FT, KB and EP brought their expertise in the design of the timeline prototype. All authors read and approved the final manuscript. All authors reviewed, corrected, and approved the final version of the manuscript. All authors agreed to be accountable for all aspects of the work in ensuring that questions related to the accuracy or integrity of any part of the work are appropriately investigated and resolved.

\section{Ethics approval and consent to participate}

The study was approved by Rennes University Hospital Ethics Committee (approval number 15.58). In accordance with the opinion of the abovementioned Ethics Committee and given the fact that no information about participants is provided in this paper, participants who participated in usability studies gave verbal consent to participate in this research.

\section{Competing interests}

The authors declare that they have no competing interests.

\section{Publisher's Note}

Springer Nature remains neutral with regard to jurisdictional claims in published maps and institutional affiliations.

\section{Author details}

${ }^{1}$ INSERM, U1099, F-35000 Rennes, France. 'Université de Rennes 1, LTSI, F-35000 Rennes, France. ${ }^{3} \mathrm{CHU}$ Rennes, F-35000 Rennes, France. ${ }^{4}$ ERIAS, INSERM U897, ISPED, Université Bordeaux, Bordeaux, France. ${ }^{5}$ Centre Régional de Pharmacovigilance, CHU Pontchaillou, Rennes, France.

\section{Received: 22 September 2017 Accepted: 1 October 2018}

\section{Published online: 19 October 2018}

\section{References}

1. WHO | Pharmacovigilance [Internet]. [cited 2017 Aug 21]. Available from: http://www.who.int/medicines/areas/quality_safety/safety_efficacy/ pharmvigi/en/
2. Murphy SN, Weber G, Mendis M, Gainer V, Chueh HC, Churchill S, et al. Serving the enterprise and beyond with informatics for integrating biology and the bedside (i2b2). J Am Med Inform Assoc. 2010;17(2):124-30.

3. Cuggia M, Garcelon N, Campillo-Gimenez B, Bernicot T, Laurent J-F, Garin E, et al. Roogle: an information retrieval engine for clinical data warehouse. Stud Health Technol Inform. 2011;169:584-8.

4. Cuggia M, Campillo-Gimenez B, Bouzille G, Besana P, Jouini W, Dufour J-C, et al. Automatic selection of clinical trials based on a semantic web approach. Stud Health Technol Inform. 2015;216:564-8.

5. Osmont M-N, Campillo-Gimenez B, Metayer L, Jantzem H, Rochefort-Morel C, Cuggia $M$, et al. Peranesthesic anaphylactic shocks: contribution of a clinical data warehouse. Therapie. 2015. p. 16. Available from: https://www. sciencedirect.com/science/article/pii/S0040595716311350.

6. Polard E, Nowak E, Happe A, Biraben A, Oger E. For the GENEPI study group. Brand name to generic substitution of antiepileptic drugs does not lead to seizure-related hospitalization: a population-based case-crossover study. Pharmacoepidemiol Drug Saf. 2015;24(11):1161-9.

7. Plaisant C, Mushlin R, Snyder A, Li J, Heller D, Shneiderman B. LifeLines: using visualization to enhance navigation and analysis of patient records. Proc AMIA Symp. 1998. p. 76-80. Available from: https://www.ncbi.nlm.nih. gov/pmc/articles/PMC2232192/.

8. Gotz D, Wang F, Perer A. A methodology for interactive mining and visual analysis of clinical event patterns using electronic health record data. J Biomed Inform. 2014;48:148-59.

9. Alexander Rind SM. VisuExplore: gaining new medical insights from visual exploration. 2010;

10. Monroe M, Meyer TE, Plaisant C, Lan R, Wongsuphasawat K, Coster TS, et al. Visualizing patterns of drug prescriptions with EventFlow: a pilot study of asthma medications in the military health system. 2013

11. Gschwandtner T, Aigner W, Kaiser K, Miksch S, Seyfang A. CareCruiser: Exploring and visualizing plans, events, and effects interactively. In: Visualization Symposium (PacificVis), 2011 IEEE Pacific; 2011. p. 43-50.

12. Shahar Y, Goren-Bar D, Boaz D, Tahan G. Distributed, intelligent, interactive visualization and exploration of time-oriented clinical data and their abstractions. Artif Intell Med. 2006;38(2):115-35.

13. Thiessard F, Mougin F, Diallo G, Jouhet V, Cossin S, Garcelon N, et al. RAVEL: retrieval and visualization in ELectronic health records. Stud Health Technol Inform. 2012;180:194-8.

14. Bostock M. D3.js - Data-Driven Documents [Internet]. [cited 2017 Aug 21]. Available from: https://d3js.org/

15. Lamy J-B, Duclos C, Hamek S, Beuscart-Zéphir M-C, Kerdelhué G, Darmoni S, et al. Towards iconic language for patient records, drug monographs, guidelines and medical search engines. Stud Health Technol Inform. 2010;160(Pt 1):156-60.

16. Nielsen J. Why you only need to test with 5 users [Internet]. 2000 [cited 2017 Aug 21]. Available from: https://www.nngroup.com/articles/why-youonly-need-to-test-with-5-users/

17. Rubin J, Chisnell D. Handbook of usability testing: how to plan, design, and conduct effective tests. Indianapolis: Wiley; 2011. p. 353.

18. ISO 9241-11:1998(en), Ergonomic requirements for office work with visual display terminals (VDTs) — Part 11: Guidance on usability [Internet]. [cited 2017 Aug 21]. Available from: https:/www.iso.org/obp/ui/\#iso:std:iso:9241:-11:ed-1:v1:en

19. Nair KM, Malaeekeh R, Schabort I, Taenzer P, Radhakrishnan A, Guenter D. A clinical decision support system for chronic pain Management in Primary Care: usability testing and its relevance. J Innov Health Inform. 2015;22(3):329-32.

20. Kamal J, Rogers P, Saltz J, Mekhjian H. Information warehouse as a tool to analyze computerized physician order entry order set utilization: opportunities for improvement. AMIA Annu Symp Proc AMIA Symp AMIA Symp. 2003. p. 336-40. Avaiblable from: https://www.ncbi.nlm.nih.gov/pmc/articles/PMC1480158/.

21. Neri PM, Pollard SE, Volk LA, Newmark LP, Varugheese M, Baxter S, et al. Usability of a novel clinician interface for genetic results. J Biomed Inform. 2012 Oct;45(5):950-7.

22. Nielsen J. Usability engineering. San Francisco: Morgan Kaufmann Publishers Inc.; 1993.

23. Olmsted-Hawala EL, Murphy ED, Hawala S, Ashenfelter KT. Think-aloud protocols: a comparison of three think-aloud protocols for use in testing data-dissemination web sites for usability. In: Proceedings of the SIGCHI Conference on Human Factors in Computing Systems [Internet]. New York: ACM; 2010. p. 2381-90. [cited 2016 Jan 6]. (CHI '10). Available from: http://doi.acm.org/10.1145/1753326.1753685.

24. Severity Ratings for Usability Problems: Article by Jakob Nielsen [Internet]. [cited 2017 Sep 18]. Available from: https://www.nngroup.com/articles/howto-rate-the-severity-of-usability-problems/ 
25. Peute LWP, de Keizer NF, Jaspers MWM. The value of retrospective and concurrent think aloud in formative usability testing of a physician data query tool. J Biomed Inform. 2015 Jun;55:1-10.

26. Brooke J. SUS: a quick and dirty usability scale. 1996

27. Lewis JR, Sauro J. The factor structure of the system usability scale. In: Proceedings of the 1st International Conference on Human Centered Design: Held As Part of $\mathrm{HCl}$ International 2009 [Internet]. Berlin: Springer-Verlag; 2009. p. 94-103. [cited 2016 Jan 6]. (HCD 09). Available from: https://doi.org/10.1007/978-3-642-02806-9_12.

28. Osmont M-N, Cuggia M, Polard E, Riou C, Balusson F, Oger E. Utilisation du PMSI pour la détection d'effets indésirables médicamenteux. Therapie. 2013 Jul 1;68(4):285-95.

29. R Development Core Team. R: a language and environment for statistical computing [internet]. Vienna: R Foundation for Statistical Computing; 2008. Available from: https://www.r-project.org/

30. Sauro J, Lewis JR. Chapter 8 - standardized usability questionnaires. In: Quantifying the user experience [internet]. Boston: Morgan Kaufmann; 2012. p. 185-240. Available from: http://www.sciencedirect.com/science/article/ pii/B9780123849687000084.

31. Martins SB, Shahar Y, Galperin M, Kaizer H, Goren-Bar D, McNaughton D, et al. Evaluation of KNAVE-II: a tool for intelligent query and exploration of patient data. Stud Health Technol Inform. 2004:107(Pt 1):648-52.

Ready to submit your research? Choose BMC and benefit from:

- fast, convenient online submission

- thorough peer review by experienced researchers in your field

- rapid publication on acceptance

- support for research data, including large and complex data types

- gold Open Access which fosters wider collaboration and increased citations

- maximum visibility for your research: over $100 \mathrm{M}$ website views per year

At BMC, research is always in progress.

Learn more biomedcentral.com/submissions 